\title{
Estrogen Modifies an Electrocommunication Signal by Altering the Electrocyte Sodium Current in an Electric Fish, Sternopygus
}

\author{
Kent D. Dunlap, M. Lynne McAnelly, and Harold H. Zakon \\ Department of Zoology and Center for Developmental Biology, University of Texas at Austin, Austin, Texas 78712
}

Many species of electric fish emit sexually dimorphic electrical signals that are used in gender recognition. In Sternopygus, mature females produce an electric organ discharge (EOD) that is higher in frequency and shorter in pulse duration than that of mature males. EOD pulse duration is determined by ion currents in the electrocytes, and androgens influence EOD pulse duration by altering the inactivation kinetics of the electrocyte sodium current. We examined whether estrogen modulates the female-specific EOD and, if so, whether it regulates EOD pulse duration by acting on the same androgen-sensitive ion current in the electrocytes. We implanted gonadectomized Sternopygus with either empty SILASTIC capsules (control), one capsule filled with estradiol-17 $\beta$ ( $E_{2}$; low dose), or three capsules of $E_{2}$ (high dose). Twelve days after implantation, $E_{2}$-treated fish had plasma $\mathrm{E}_{2}$ levels $\sim 3.3$-fold (low dose) or $\sim 7.1$-fold (high dose) higher than controls. After implantation, both $\mathrm{E}_{2}$-treated groups had higher EOD frequency and shorter EOD pulse duration than controls and their own preimplantation values. Through immunocytochemistry, we identified immunoreactive estrogen receptors in the nuclei of electrocytes, indicating that these cells are directly responsive to estrogen. In addition, voltage-clamp studies showed that $E_{2}$ affected the electrocyte ion currents kinetics: the sodium inactivation time constant was significantly lower in $\mathrm{E}_{2}$-treated fish than in controls. Thus, sexual dimorphism in the electrocommunication signal results, at least in part, from estrogens and androgens acting in opposite directions on the same ion current in the electrocytes.

Key words: estrogen; sodium current; electric fish; estrogen receptor; electric organ; Sternopygus
In most vertebrates, steroids have potent influences on the expression of sexually dimorphic reproductive behaviors such as courtship and mating (Kelley, 1988). Many studies have shown that regions of the nervous system and the effector organs that control reproductive behaviors have specific receptors for sex steroids, and more recently researchers have found that steroids can induce changes in the overall extracellular electrical activity in brain nuclei (Becker et al., 1992). Our understanding, however, of how steroids alter the electrical properties in specific, behaviorally relevant cells is still limited.

Recent studies on the sexually dimorphic electrocommunication signal of a weakly electric fish, Sternopygus, have demonstrated that androgens alter specific ionic conductances in excitable cells involved in reproductive behavior (Ferrari and Zakon, 1993; Zakon, 1993; Ferrari et al., 1995). This has been possible because the neural circuits and effector cells underlying this behavior are relatively simple and discrete (Bullock and Heiligenberg, 1986), and some cells, the electrocytes of the electric organ, are large and accessible for recording changes in membrane properties by voltage clamp (Ferrari and Zakon, 1993; Ferrari et al., 1995; McAnelly and Zakon, 1996).

In Sternopygus, males and females recognize each other through sexually dimorphic electric discharges emitted from their electric organs: males discharge at low frequencies $(50-80 \mathrm{~Hz})$ and fe-

Received Oct. 2, 1996; revised Jan. 27, 1997; accepted Jan. 31, 1997.

This work was supported by National Institutes of Health through a fellowship to K. Dunlap (1 F32 NS09633-01A1) and a grant to H. Zakon (RO1-NS25513). We thank D. Breckenridge and P. Thomas at the University of Texas Marine Science Institute for conducting the steroid assays, J. Schaefer for technical assistance, Y. Lu for fish care, and Abbott Laboratories for providing the estrogen receptor antibody.

Correspondence should be addressed to Kent Dunlap, Department of Zoology, University of Texas, Austin TX 78712.

Copyright (C) 1997 Society for Neuroscience $0270-6474 / 97 / 172869-07 \$ 05.00 / 0$ males at high frequencies (120-200 Hz) (Hopkins, 1974; Zakon et al., 1991a,b). The frequency of the electric organ discharge (EOD) is regulated by the pacemaker nucleus in the hindbrain, and the EOD pulse duration is determined by ion currents in the electrocytes of the electric organ. Although EOD frequency and pulse duration are controlled independently, they are correlated negatively (Mills and Zakon, 1987, 1991). Thus, females have a shorter pulse duration than males.

The male-specific EOD is modulated by androgens. EOD frequency varies inversely with plasma androgens in males during the natural breeding season (Zakon et al., 1991a,b), and in the laboratory, exogenous androgen [testosterone $(\mathrm{T})$ and dihydrotestosterone] treatment lowers EOD frequency and broadens pulse duration (Meyer, 1983, 1984; Zakon et al., 1991a,b). It is not known how androgens alter the medullary neurons that control EOD frequency, but recent voltage-clamp studies on electrocytes show that androgens increase EOD pulse duration by causing the sodium current to inactivate more slowly (Ferrari and Zakon, 1993; Zakon, 1993; Ferrari et al., 1995).

Although these studies documented how steroids modulate the male-specific EOD, little is known about mechanisms generating the female-specific EOD. At least three possibilities exist. (1) The female EOD is steroid-independent, and sexual dimorphism is generated strictly through androgens lowering EOD frequency in males; (2) females secrete hormones (e.g., estrogen) that act to reduce plasma $\mathrm{T}$ levels and thereby raise EOD frequency; or (3) females secrete hormones that act directly but in opposite directions on the androgen-sensitive tissues. We tested whether estradiol is effective in generating the female-specific EOD and, if so, whether it acts on the same tissues and through the same cellular mechanisms as androgens. 


\section{MATERIALS AND METHODS}

Overview. Adult Sternopygus were gonadectomized and implanted with estrogen-filled capsules or empty capsules. EOD frequency and pulse duration were recorded before implantation and for $12 \mathrm{~d}$ after implantation. Fish were then bled, and plasma estradiol and $\mathrm{T}$ concentrations were measured. Twelve to fifteen days after implantation, the sodium current kinetics in the electrocytes was determined in a subset of fish using voltage clamp. These fish were later treated again with estrogen (or nothing), and estrogen receptors (ERs) were identified in their electric organs and livers using immunocytochemistry. Details of these procedures are given below.

Animals and housing conditions. Twenty-three adult $(>28 \mathrm{~cm}$ snout to tail) Sternopygus were obtained commercially and housed in individual tanks that were part of a circulating 400 gallon system. They were fed earthworms every 2-3 d. The photoperiod (12 hr light/dark), water temperature $\left(27.3 \pm 0.2^{\circ} \mathrm{C}\right)$, and water conductivity $(1100 \mu \mathrm{S})$ were held constant throughout the experiment.

Gonadectomy and hormone implantation. Fish were anesthetized in 0.075\% 2-phenoxyethanol (P-1126, Sigma, St. Louis, MO). Gonads and accessory ducts were removed through a $\sim 2 \mathrm{~cm}$ incision in the posterior ventrolateral body wall. The incision was closed with 5-7 sutures (5.0 surgical silk), the wound was treated with antibiotic, and the fish was allowed to recover in its home tank.

Three to six weeks after gonadectomy, the fish were divided into three treatment groups: low dose, high dose, and control. Fish of both sexes were used based on the observation that males and females respond similarly to exogenous steroid treatment (Meyer, 1983). The availability of female fish was limited, and thus not all groups contained equal numbers of males and females. As much as possible, fish were distributed equally to treatment groups according to sex, body size, and EOD frequency. The low-dose group (four males, two females; mean preimplantation EOD frequency $=93.7 \pm 4.6)$ received one capsule filled with estradiol-17 $\beta\left(E_{2}\right)$ (E-8875, Sigma), the high dose group (five males, four females; preimplantation EOD frequency $=93.6 \pm 6.9)$ received three filled capsules, and the controls (five males, four females; preimplantation EOD frequency $=92.7 \pm 4.6)$ received three empty capsules. The capsule consisted of a SILASTIC tube $(0.64 \mathrm{~mm}$ inner diameter $\times 1.20$ $\mathrm{mm}$ outer diameter $\times 8 \mathrm{~mm}$ length; Dow Corning model no. 602-155) sealed at each end with SILASTIC adhesive. Each filled capsule contained $\sim 4 \mathrm{mg} \mathrm{E}_{2}$, resulting in a total dosage of $\sim 50 \mu \mathrm{g} / \mathrm{gm}$ body weight (low dose) or $\sim 150 \mu \mathrm{g} / \mathrm{gm}$ body weight (high dose). To implant the capsules, we anesthetized the fish as above, punctured a small hole in the lateral body wall using an 18 gauge needle, and inserted the capsules into the anterior peritoneal cavity.

EOD measurements. EOD frequency was recorded daily for $4 \mathrm{~d}$ before implantation and for $12 \mathrm{~d}$ after implantation. EOD pulse duration was recorded three times: once immediately before implantation and again on days 6 and 12 after implantation.

EOD frequency and pulse duration were measured using methods similar to those reported in previous publications (Mills and Zakon, 1987, 1991). Briefly, EOD was measured through two bare wires held within 2 $\mathrm{cm}$ of the fish's tail. The signal was amplified by a Grass P15 AC-coupled amplifier, and the EOD frequency was read on a FLUKE voltmeter in the frequency counter mode. To measure EOD pulse duration, the output of the amplifier was fed into a World Precision Instruments Universal Signal Manifold, stored on a Standard 286 AT clone, and analyzed using the software SPIKE. For each fish, we measured the pulse duration of 4-6 cycles. All EOD measurements were standardized to a temperature of 27.3 ${ }^{\circ} \mathrm{C}$ using a $Q_{10}$ of 1.5 (Enger and Szabo, 1968).

Blood collection and hormone analysis. Twelve days after implantation, fish were anesthetized and bled from the caudal sinus into heparinized syringes fitted with 25 gauge needles. Blood was stored in heparinized tubes on ice until it was centrifuged, and plasma was stored at $-25^{\circ} \mathrm{C}$.

Plasma $\mathrm{E}_{2}$ concentrations and $\mathrm{T}$ were determined by radioimmunoassay in the laboratory of P. Thomas, University of Texas Marine Science Institute. This assay was validated previously for Sternopygus in the Thomas laboratory (Zakon et al., 1990). Each steroid was extracted separately from 100-200 $\mu \mathrm{l}$ of plasma with a 70:30 mixture of hexane and ethyl acetate. The aqueous portion was removed, the solvent layer was evaporated with nitrogen, and the hormone residue was reconstituted in phosphate buffer. Each sample was incubated overnight at $4^{\circ} \mathrm{C}$ with the appropriate antiserum (Radioassay Systems Laboratory) and tritiated steroid. Based on previous studies, the estradiol antiserum cross-reacts $22.3 \%$ with 16 -ketoestradiol, $2.5 \%$ with estriol, and $1.3 \%$ with estrone, and the $\mathrm{T}$ antibody reacts $28.0 \%$ with dihydrotestosterone and $17.0 \%$ with 11-ketotestosterone (Zakon et al., 1990). The unbound fraction was removed by charcoal and centrifugation, and the bound fraction was counted in a scintillation counter.

Voltage clamp of electrocytes. Voltage clamp was conducted $12-15 \mathrm{~d}$ after implantation on a subset of the above experimental fish. Five high-dose fish (two males, three females) and five control fish (two males, three females) were selected based on the similarity of their preimplantation EOD frequency and pulse duration. We chose to examine only high-dose fish, because in this group the estrogen effect on EOD remained pronounced until day 12 (see Fig. 1). The sodium current was recorded from two to eight electrocytes in each fish.

The sodium current in electrocytes was measured by voltage clamp using the technique described by Ferrari and Zakon (1993). The posterior $1-2 \mathrm{~cm}$ of the tail was amputated and placed in saline containing $110 \mathrm{~mm}$ $\mathrm{NaCl}, 2 \mathrm{~mm} \mathrm{KCl}, 4 \mathrm{~mm} \mathrm{CaCl}_{2}, 1 \mathrm{~mm} \mathrm{MgCl}_{2}, 5 \mathrm{~mm}$ HEPES, and $3 \mathrm{~mm}$ glucose. The skin was dissected carefully from the underlying electric organ, exposing the most superficial electrocytes. To increase the space constant of the electrocytes in the recording chamber, the chloride leak was blocked by replacing the $\mathrm{NaCl}$ with sodium methyl sulfate in the saline. We also added $40 \mathrm{~mm}$ tetraethylammonium (TEA) and $2 \mathrm{~mm} \mathrm{CsCl}$ to the bathing solution to block the outward rectifying potassium current and the inward rectifying potassium current, respectively.

Two microelectrodes filled with $3 \mathrm{M} \mathrm{KCl}$ were inserted into electrocytes near the active (posterior) face of the cell. One electrode was used to apply current and the other to record consequent voltage changes. Voltage pulses were generated by pCLAMP software and delivered to an Axoclamp 2-A amplifier via a TL-1 DMA interface (Axon Instruments, Foster City, CA), and the membrane current was sampled at $20 \mathrm{kHz}$. The initial recordings were collected while the potassium currents were blocked pharmacologically with TEA and CsCl. We then used a depolarizing prepulse to inactivate the sodium current, leaving only the remaining leakage current. This second current was then subtracted from the first recording to yield the isolated sodium current. The sodium current inactivation time constant (tau) was calculated from the pCLAMP curve fitting procedure using a single exponential decay.

Immunocytochemistry. To aid in localizing ERs in Sternopygus, we treated fish with estradiol before immunocytochemistry. Exogenous estrogen increases ER mRNA expression in the brain (Salbert et al., 1993) and liver (Pakdel et al., 1991) of another teleost, the rainbow trout. We reimplanted control and $\mathrm{E}_{2}$-treated Sternopygus from the above experiments with empty or $\mathrm{E}_{2}$-filled capsules $(1 \mathrm{~cm}$; unsealed at one end), respectively. Twelve to sixteen hours later, the fish were anesthetized deeply, and the posterior $2 \mathrm{~cm}$ of the tail and the liver were removed from the fish. These tissues were frozen immediately $\left(-80^{\circ} \mathrm{C}\right)$ in isopentane. Tissues were cut in cross section $(15 \mu \mathrm{m})$ on a freezing cryotome, air-dried onto the slide, and fixed in $4 \%$ paraformaldehyde $\left(4^{\circ} \mathrm{C}\right)$ for 1 min. Unless indicated otherwise, all solutions were used at $4^{\circ} \mathrm{C}$. Sections were then rinsed $(3 \times 5 \mathrm{~min})$ in PBS $(0.1 \mathrm{M})$, placed in cold methanol $\left(-20^{\circ} \mathrm{C}\right)$ for $4 \mathrm{~min}$ followed by cold acetone $\left(-20^{\circ} \mathrm{C}\right)$ for $2 \mathrm{~min}$, and rinsed in PBS $(2 \times 10 \mathrm{~min})$ again. To reduce nonspecific binding, sections were preincubated for $1 \mathrm{hr}$ in PBS containing goat serum (GS; 10\%) and bovine serum albumin (BSA; $0.2 \%$, Sigma A-7906). The primary antibody (anti-ER, H222, Abbott Laboratories, Irving, TX; $5.6 \mu \mathrm{g} / \mathrm{ml}$ in GS/BSA/ PBS) was placed on the slides, and the sections were incubated for 42-48 $\mathrm{hr}$ in a humidity chamber at $4^{\circ} \mathrm{C}$. Slides were then rinsed (GS/BSA/PBS, $3 \times 5 \mathrm{~min}$ ), incubated in fluorescein isothiocyanate-conjugated secondary antibody (Cappell 55513; 1:200) for $1 \mathrm{hr}$, and rinsed again (PBS; $2 \times 10$ min). To counterstain cell nuclei, sections were placed in propidium iodide (Molecular Probes P-3566, $0.5 \mu \mathrm{g} / \mathrm{ml}$ ) for $5 \mathrm{~min}$ at room temperature and rinsed in PBS $(2 \times 5 \mathrm{~min})$. Slides were then mounted in gel (Biomeda, M01) and viewed with a Nikon Diaphot fluorescence microscope.

We ran two sets of controls to confirm that this procedure was effective and specific in identifying ERs. First, as a positive control, liver was run in parallel with electric organ. Teleost liver is rich in ERs because of the estrogen regulation of hepatic vitellogenin synthesis (Fostier et al., 1983; Pakdel et al., 1991). Second, every other section was incubated without primary antibody, enabling us to recognize the nonspecific staining of the secondary antibody.

Statistics. The effects of treatment (estrogen vs control), gender (male vs female), and time (the repeated measure) on EOD frequency and pulse duration were determined by two-way, repeated measures ANOVA. Treatment and gender effects on postimplantation hormone level and sodium current inactivation time constant (tau) were determined by two-way ANOVA. Correlations among variables were deter- 
Table 1. Plasma steroid concentrations in gonadectomized Sternopygus implanted with an empty capsule (control), one capsule of estradiol (low dose), or three capsules of estradiol (high dose)

\begin{tabular}{llll} 
& $\begin{array}{l}\text { Control } \\
n=9\end{array}$ & $\begin{array}{l}\text { Low dose } \\
n=6\end{array}$ & $\begin{array}{l}\text { High dose } \\
n=9\end{array}$ \\
\hline Estradiol-17 $\beta(\mathrm{ng} / \mathrm{ml})$ & $1.71(0.43)$ & $5.71(1.23)^{*}$ & $12.22(3.71)^{*}$ \\
Testosterone $(\mathrm{ng} / \mathrm{ml})$ & $0.41(0.05)$ & $0.35(0.35)$ & $0.32(0.05)$ \\
\hline
\end{tabular}

Data are presented as means (SEM).

*Indicates a significant difference between treatment groups.

mined using product-moment regression. Differences between groups in the intercept of the regression were determined by analysis of covariance (ANCOVA). $p$ values $<0.05$ were considered significant.

\section{RESULTS}

\section{Hormone levels}

Implants raised plasma $\mathrm{E}_{2}$ levels in a dose-dependent manner (Table 1). At $12 \mathrm{~d}$ postimplantation, treatment elevated plasma $\mathrm{E}_{2}$ $\sim 3.3$-fold over controls in the low-dose group and $\sim 7.1$-fold in the high-dose group $(F=7.29 ; p<0.005)$. Surprisingly high $\mathrm{E}_{2}$ levels were found in control fish; however, postmortem dissection failed to show any apparent residual gonadal tissue. In addition, in the control group, females did not have higher $\mathrm{E}_{2}$ levels than males, as one would expect if actively secreting gonadal tissue persisted after gonadectomy. Thus, it seems that in Sternopygus, as in other teleosts (Fostier et al., 1983), extra-gonadal tissue (e.g., liver, kidney) produces considerable levels of estradiol.

Because previous studies showed that androgens have potent effects on the EOD, we measured post-treatment $T$ levels to test the possibility that $\mathrm{E}_{2}$ exerts its effect indirectly by lowering $\mathrm{T}$ levels. Although both $\mathrm{E}_{2}$-treated groups had lower $\mathrm{T}$ levels than controls, the difference was not statistically significant (Table 1) $(p>0.05)$. There were also no differences in T levels $(p>0.05)$ between males and females.

Fish in both $\mathrm{E}_{2}$-treated groups had higher circulating $\mathrm{E}_{2}$ levels than the field-captured female Sternopygus reported by Zakon et al. (1991b); however, this field study likely underestimated the maximal breeding levels of $E_{2}$, because few of the females were in the late stages of oocyte maturation. In addition, data from the field study and the present study are not wholly comparable because of differences in the assay protocols. $E_{2}$ was separated chromatographically from other steroids before radioimmunoassay in the field study but not in present study. Steroid values presented here likely overestimate the the concentration of $E_{2}$ in the plasma attributable to cross-reactivity of the antiserum with other estrogens (e.g., 16-ketoestradiol, estriol, and estrone; see Materials and Methods).

\section{EOD frequency and pulse duration}

Experimental groups had equivalent EOD frequency and pulse duration before implantation $(F=0.10 ; p>0.05)$, but $\mathrm{E}_{2}$ treatment significantly increased EOD frequency $(F=1.86 ; p<0.01)$ and decreased EOD pulse duration (Fig. 1$)(F=3.64 ; p<0.01)$. Control fish were not affected significantly by implantation of an empty capsule $(F=0.07 ; p>0.05)$. $\mathrm{E}_{2}$-treated fish had significantly higher EOD frequency $(F=2.38 ; p<0.05)$ than controls beginning $24 \mathrm{hr}$ post-treatment and continuing for the next $11 \mathrm{~d}$ (Fig. 1). Low-dose and high-dose groups showed equivalent rates of increase in EOD frequency through the first week, after which the low-dose group appeared to plateau or decrease slightly. $\mathrm{E}_{2}$ treatment decreased EOD pulse duration significantly by day 6
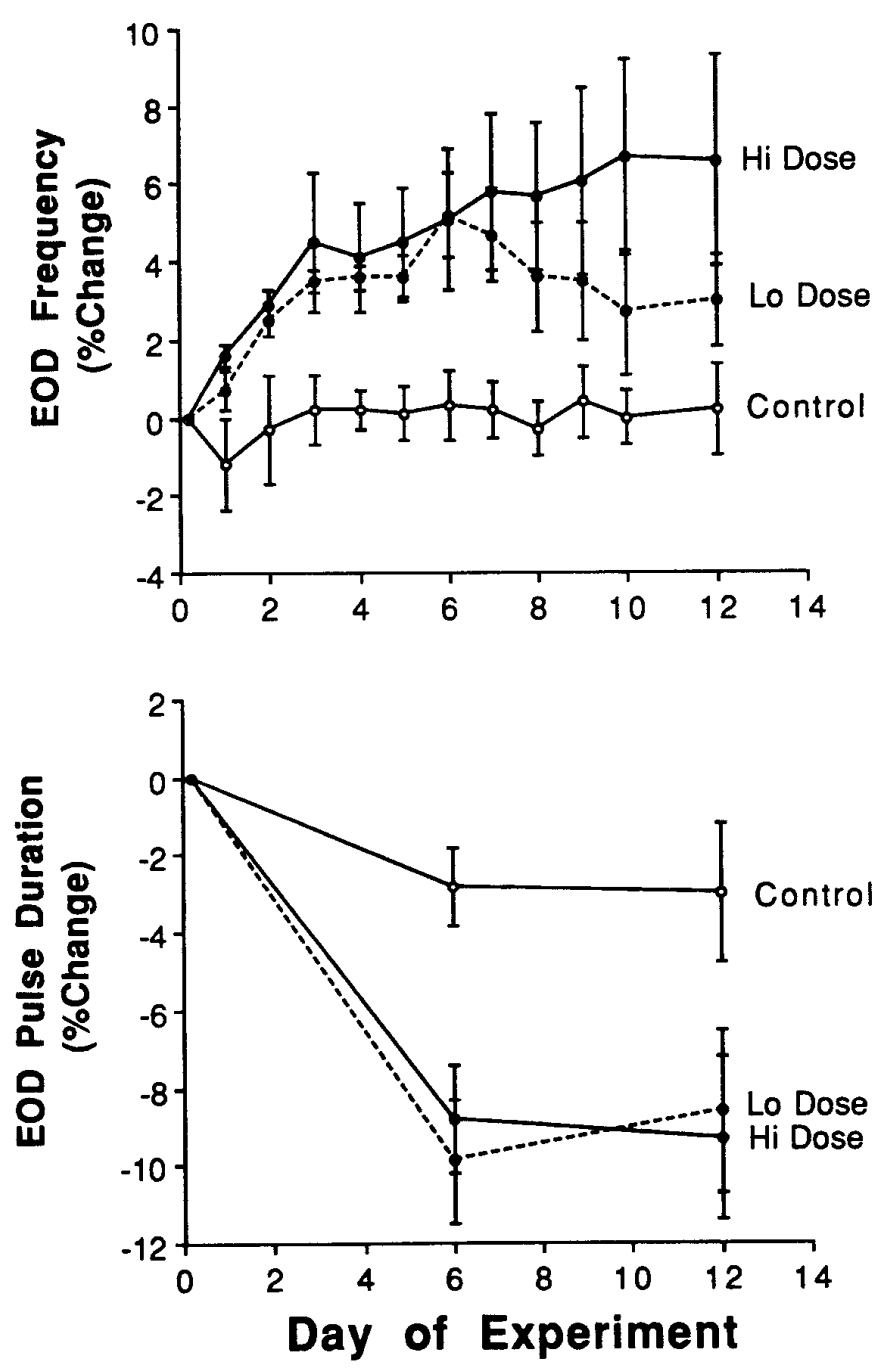

Figure 1. Percentage changes in the electric organ discharge frequency (EOD Frequency) and pulse duration in gonadectomized Sternopygus implanted with an empty capsule (Control; open circle; $n=9$ ), one capsule filled with estradiol (Lo dose; closed circle and dotted line; $n=6$ ), or three estradiol-filled capsules (Hi dose; closed circle and solid line; $n=9$ ). Data are presented as means, with vertical bars representing SEM. See Results for mean preimplantation values.

$(F=3.94 ; p<0.001)$, and the effect did not differ between dosage groups.

There were no differences between males and females in EOD parameters in either the control or $\mathrm{E}_{2}$-treated group $(p>0.05)$.

\section{Sodium current kinetics in electrocytes}

Accompanying the increase in EOD frequency and the decrease in pulse duration, the electrocyte sodium current inactivated more quickly in $E_{2}$-treated fish than in control fish (Figs. 2, 3). This is evidenced by a significantly lower mean inactivation time constant (tau) in $\mathrm{E}_{2}$-treated fish (Fig. 3) $(F=6.9 ; p<0.05)$. Tau was usually very consistent among electrocytes within a single fish, with coefficients of variation ranging from 3.5 to 16.5. Mean tau correlated negatively with EOD frequency $\left(r^{2}=-0.81 ; p<0.01\right)$ and positively with pulse duration $\left(r^{2}=0.87 ; p<0.005\right)$. Neither the slope nor the intercept of these regression lines differed significantly between control and $\mathrm{E}_{2}$-treated fish (ANCOVA; $F=$ $0.4 ; p>0.5)$. Tau in electrocytes from males and females did not differ in either the control or $\mathrm{E}_{2}$-treated fish $(F=0.86 ; p>0.05)$. 


\section{A. Estrogen-treated}

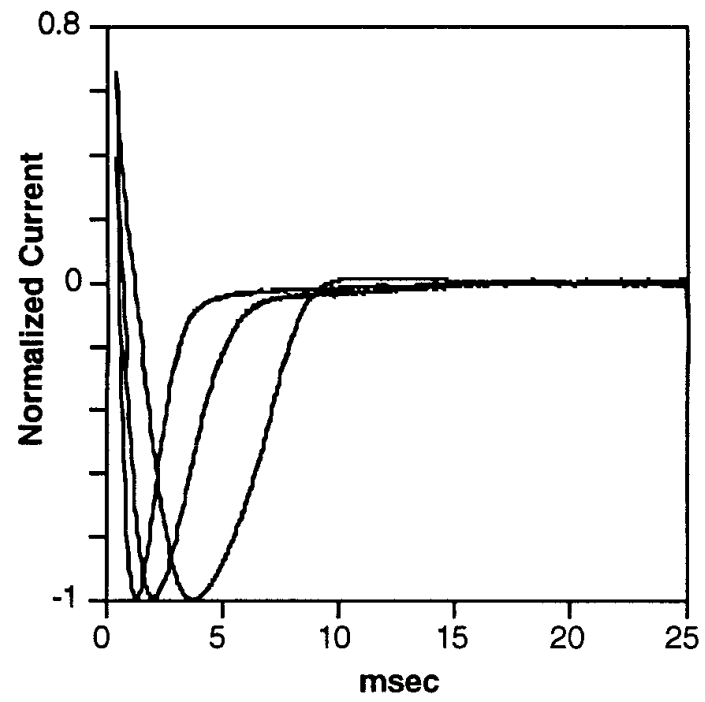

B. Control

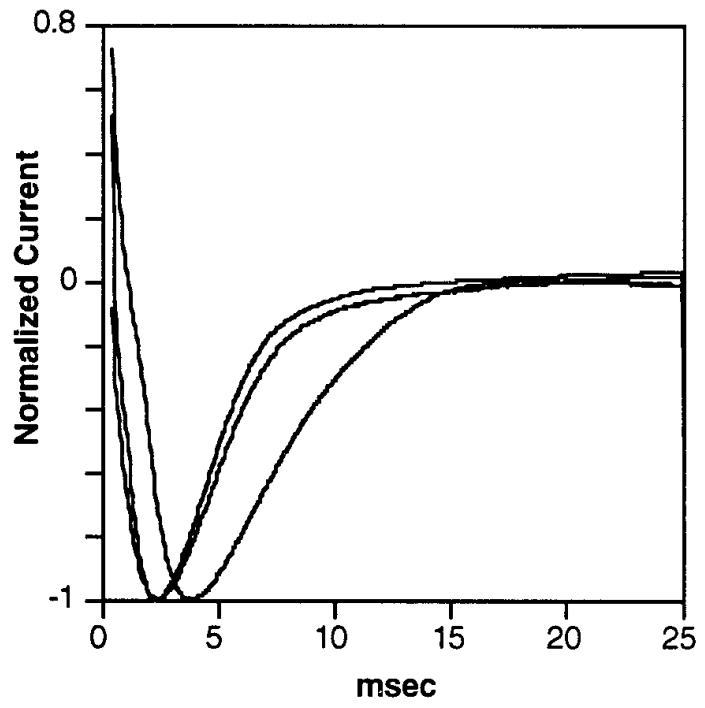

Figure 2. Representative voltage-clamp traces of the peak electrocyte sodium current from $(A)$ three fish implanted with $\mathrm{E}_{2}$-filled capsules and $(B)$ three control fish implanted with empty capsules. Holding potential ranged from -73 to $-100 \mathrm{mV}$. The membrane potential was stepped in 5 $\mathrm{mV}$ increments from $40 \mathrm{mV}$ below holding potential to $180 \mathrm{mV}$ above. Maximum sodium current amplitude was normalized to -1.0. Inactivation time constants (tau) in milliseconds for fish in this figure were $0.710,0.635$, and 1.206 for estrogen-treated fish and 2.209, 2.395, and 3.900 for control fish.

The sodium current appeared to activate more rapidly in $E_{2}$ treated fish than in control fish (Fig. 2), but this difference was not quantified.

\section{Immunocytochemistry}

Immunoreactive ERs (ir-ERs) were found in both the electric organ and liver of Sternopygus (Fig. 4). In both tissues, the density of immunoreactive nuclei appeared greater in $E_{2}$-treated than
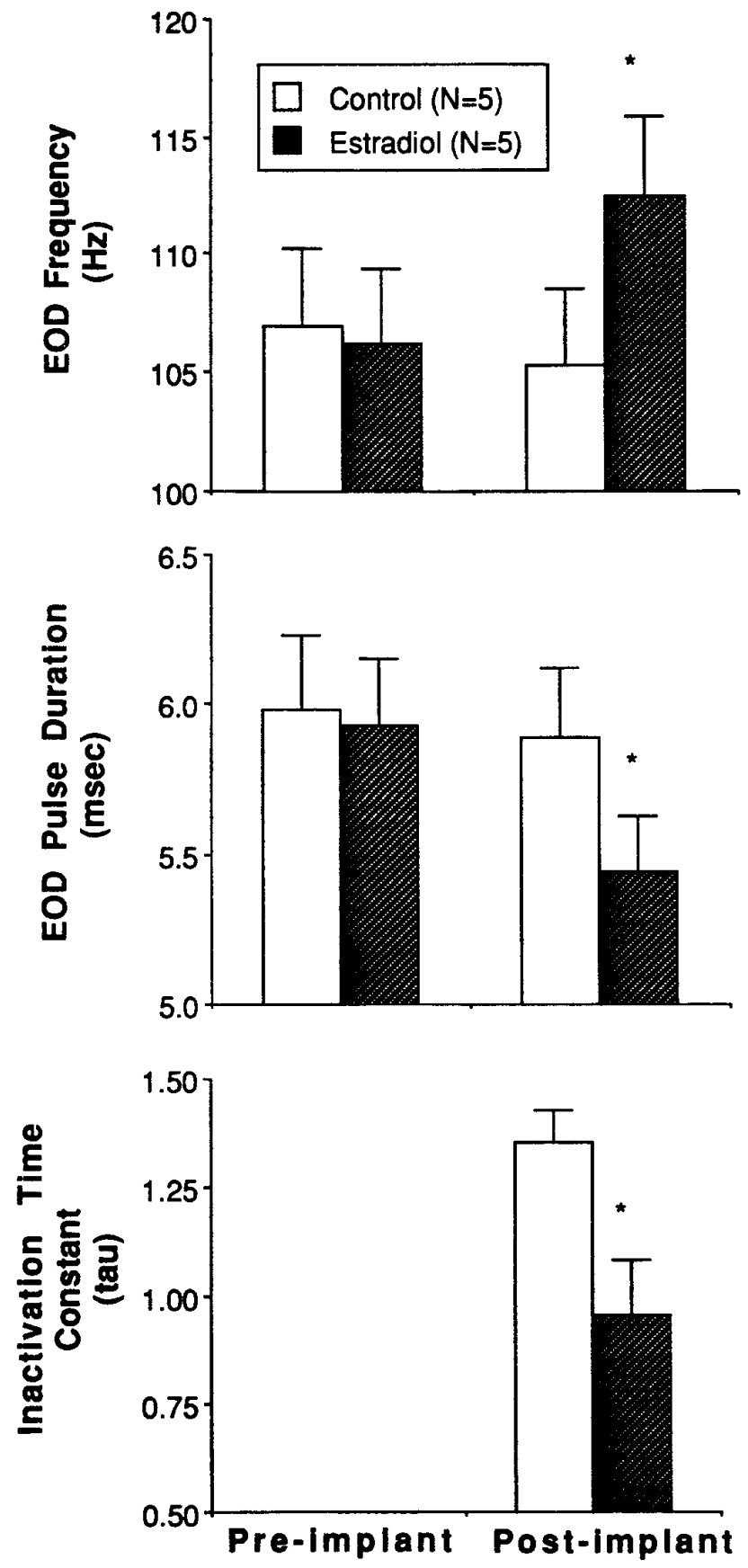

Figure 3. Pre- and postimplantation values of EOD frequency, pulse duration, and sodium current inactivation time constants (tau) in the fish used in voltage-clamp study. Control fish $(n=5)$ were implanted with an empty capsule, and $\mathrm{E}_{2}$-treated fish $(n=5)$ were implanted with three $\mathrm{E}_{2}$-filled capsules. No preimplantation values of tau were collected. Data are presented as means, and vertical bars represent SEM. Asterisks indicate significant differences between control and $\mathrm{E}_{2}$-treated groups.

control fish, although this difference was more pronounced in liver than in electric organ. In the multinucleated electrocytes of the electric organ, labeling was consistently confined to nuclei, which are located at the extreme periphery of the cells. Based on an estimate of the density of ir-ER-positive nuclei in tail cross sections, it appeared that most electrocytes $(>90 \%)$ contained nuclei with ir-ER, but a low proportion $(\sim 25 \%)$ of all electrocyte nuclei were ir-ER positive (compare Fig. $4, A$ and $B$ ). Nuclei of satellite 

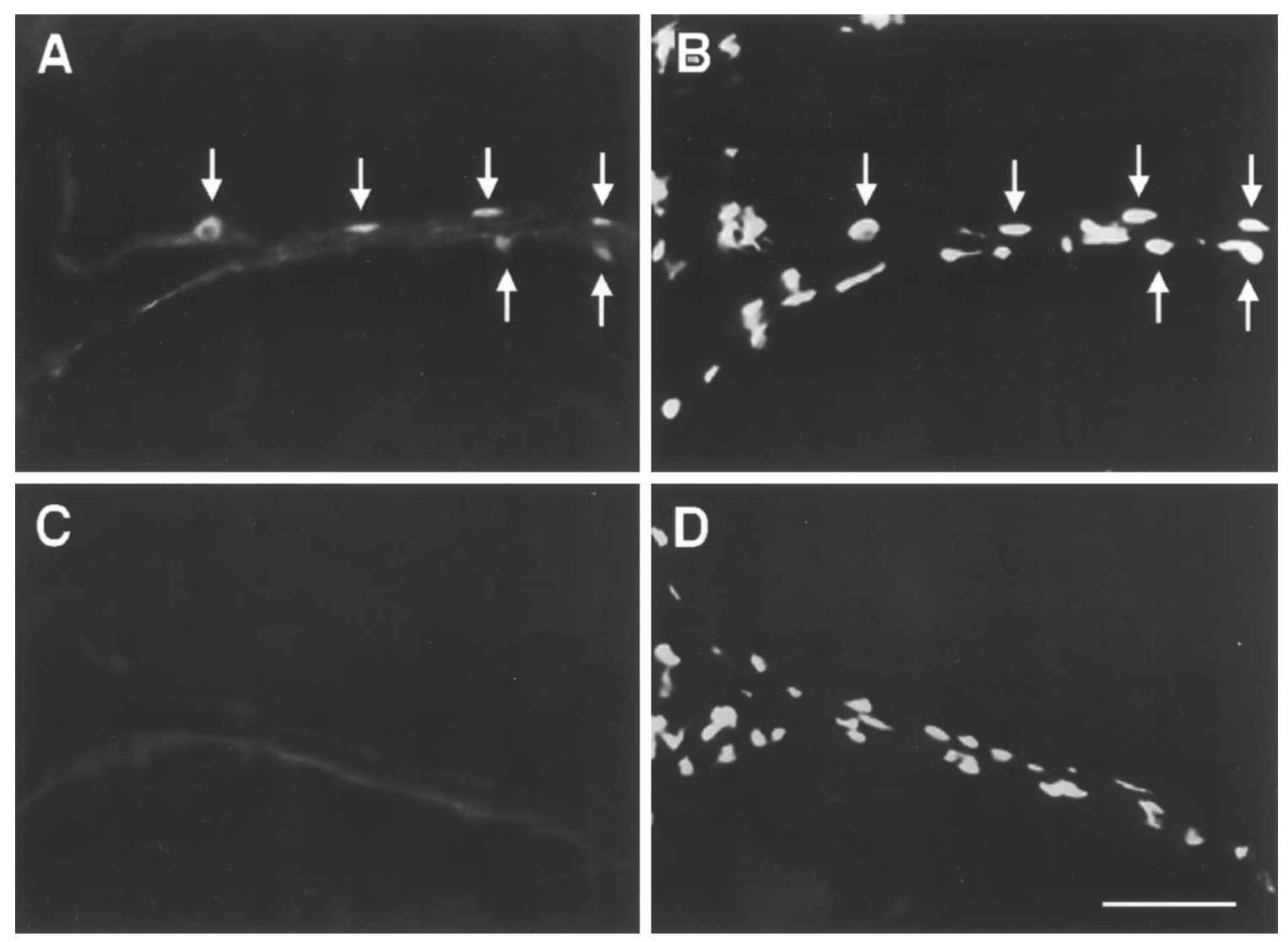

Figure 4. Immunocytochemical localization of ERs in the electric organ of Sternopygus. The fish was implanted with $\mathrm{E}_{2}$, and the tail was cut in cross section. Each photograph shows the intersection of two adjacent electrocytes. A, Section stained with antibody to ER (H222). Arrows indicate positively stained nuclei located at the periphery of adjacent cells. $B$, Same section as in $A$ stained with propidium iodide to show all nuclei present. Arrows point to nuclei stained in $A$. Note that not all nuclei stain positively with H222. $C$, Control section incubated without primary antibody to show nonspecific staining of the secondary antibody. $D$, Same section as in $C$ stained with propidium iodide. Scale bar, $50 \mu \mathrm{m}$.

cells, which are located adjacent to the electrocyte nuclei in the interstices of the electrocytes, did not show ir-ER. Labeling was also absent from all other tissue in the tail cross section (spinal cord, nerve, vertebral cartilage, blood, skin, muscle) and extremely rare in sections incubated without primary antibody (Fig. $4 C$ ). Males and females did not appear to differ in the abundance or distribution of ir-ER.

\section{DISCUSSION}

Our study shows that $\mathrm{E}_{2}$ administered to gonadectomized adult Sternopygus induces changes in the electrocommunication signal similar to those seen in maturing females and opposite to those in maturing males and androgen-treated fish. Specifically, $\mathrm{E}_{2}$ increases EOD frequency and decreases EOD pulse duration. Given the independent control of these two EOD parameters (Mills and Zakon, 1987, 1991), we believe that $\mathrm{E}_{2}$ acts both on the brain (presumably on pacemaker neurons in the hindbrain) and on the electrocytes of the electric organ. The $\mathrm{E}_{2}$-induced changes in the EOD are accompanied by an increase in the inactivation rate of the electrocyte sodium current, which is the most important determinant of the EOD pulse duration. Together with previous studies on androgens in Sternopygus, our study demonstrates that sexual dimorphism in the communication signal in this species is attributable, at least in part, to different sex steroids exerting opposite influences on the same ion channels in the electric organ.

\section{Estrogen as a modulator of the EOD}

Several lines of evidence indicate that estradiol acts as an endogenous modulator of the Sternopygus electrocommunication signal. We demonstrate that tonically released $\mathrm{E}_{2}$ is effective in modifying the EOD in gonadectomized Sternopygus (Fig. 1). Meyer (1983) first reported that $E_{2}$ increased EOD frequency, but this result was based on high doses of $E_{2}$ injected intraperitoneally, and the resulting plasma steroid concentrations were not measured. Our hormonal manipulation using implants more likely mimics the gradual rise in estrogens during natural ovarian recrudescence and produces concentrations that are within the physiological range of breeding female teleost fish (Fostier et al., 1983).

Second, Sternopygus electrocytes, which emit the EOD and regulate its pulse duration, contain nuclei that are immunoreactive for the ER (Fig. 4). In these multinucleated cells, a relatively low proportion of the nuclei label, but most electrocytes appear to contain nuclei with ERs. This indicates that electrocytes are targets for endogenous estrogen and that their genomic activity is likely subject to estrogen modulation. Electrocyte nuclei also contain immunoreactive androgen receptors (Gustavson et al., 1994). Future studies co-labeling with antibodies to estrogen and androgen receptors will be able to determine whether these distinct receptors are present in the same individual nuclei or are partitioned into separate populations of nuclei within the electrocyte. We did not examine ER 
expression in the brain, but our demonstration that estrogen affects EOD frequency suggests that the pacemaker nucleus or the prepacemaker nucleus (the only major input to the pacemaker nucleus) contains ERs. Androgen receptors are located in the prepacemaker nucleus but have not been observed in the pacemaker nucleus (Gustavson et al., 1994).

Finally, we show that estrogen effects are independent of androgen modulation of the EOD signal. Females of many fish species, including Sternopygus, have high levels of T (Fostier et al., 1983; Zakon et al., 1991b), and $\mathrm{E}_{2}$ could conceivably increase EOD frequency and shorten EOD pulse duration by suppressing the production of androgens. We show that this is unlikely because the $\mathrm{E}_{2}$-induced changes in the EOD were not accompanied by a significant decrease in $\mathrm{T}$ (Table 1 ).

Estradiol seems less potent than androgens in affecting the Sternopygus EOD. The low-dose $\mathrm{E}_{2}$-implanted group showed a $\sim 5 \%$ increase in EOD frequency and a $\sim 9 \%$ decrease in pulse duration, whereas a similar dosage of dihydrotestosterone produces a $\sim 40 \%$ decrease in EOD frequency and a $\sim 15 \%$ decrease in pulse duration (Mills and Zakon, 1991; Ferrari et al., 1995). Moreover, under field conditions, mature females typically have EOD frequencies that are $\sim 20-40 \%$ higher than juveniles (Zakon et al., 1991b). The relatively small effect of exogenous estradiol in individually housed, gonadectomized fish in the laboratory indicates that other ovarian compounds or external (e.g., social) signals are probably necessary to induce the full expression of the female-specific EOD. For example, prostaglandins produced by the maturing oocytes in the ovary are potent regulators of female reproductive behavior in other teleosts with external fertilization (Stacey, 1976; Liley and Stacey, 1983; Kobayashi and Stacey, 1993). In addition, the history and context of social interaction can influence the extent to which female fish exhibit reproductive behaviors (Liley and Wishlow, 1974; Lam, 1983).

Sternopygus seem to respond to estrogen differently than the other well studied species of neotropical electric fish (order Gymnotiformes). In contrast to Sternopygus, Apteronotus leptorhynchus (Meyer et al., 1987; Schaefer and Zakon, 1996) lower their EOD frequency in response to estradiol implantation. Preliminary evidence suggests that Apteronotus albifrons and Eigenmannia sp. also lower their EOD frequency after estradiol treatment (K. Dunlap, unpublished observations). Sternopygus is the most primitive of all gymnotiforms (Alves-Gomes et al., 1995), and thus it appears that there was a reversal in the behavioral response to estrogen relatively early in gymnotiform evolution.

\section{Mechanism of estrogen effects on electrocyte biophysical properties}

In Sternopygus, $\mathrm{E}_{2}$ increased the rate of sodium current inactivation in electrocytes (Figs. 2, 3). To our knowledge, this is the first report of estrogen influencing sodium current kinetics. Other studies, however, have shown that estrogen modifies sodium current densities in the myometrium (Boyle et al., 1987; Inoue and Sperelakis, 1991) and potassium and/or calcium currents in smooth muscle of the bladder (Yasay et al., 1995), arteries (White et al., 1995), and myometrium (Erulkar et al., 1994; Yamamoto, 1995), and in neurons of the hippocampus (Joels and Karst, 1995), hypothalamus (Lagrange et al., 1995), and neostriatum (Mermelstein et al., 1996). In a case that seems analogous to the opposing actions of estrogen and androgen on electrocytes, Erulkar et al. (1994) showed that estrogen and progesterone affect the myometrial potassium channel kinetics in opposite ways: estradiol decreases whereas progesterone increases the time constant of decay of the outward potassium current.

The precise mechanisms by which estrogen and androgen exert opposite effects on electrocyte sodium currents are still unknown, but recent studies allow us to eliminate certain possibilities and propose a hypothesis. Steroids could achieve opposite effects by differentially influencing the level of sodium channel phosphorylation. Protein kinase-induced phosphorylation alters sodium channel kinetics in brain cells (Numann et al., 1991), but we recently reported that activating protein kinase A with a cAMP analog (8-bromo-cAMP) does not influence the inactivation kinetics of electrocyte sodium channels in Sternopygus (McAnelly and Zakon, 1996). Thus, steroids probably do not operate through this pathway, although other phosphorylation pathways have not been tested.

More likely, estrogens and androgens differentially regulate the expression of multiple forms of the sodium channel. In mammals, the sodium channel exists in at least two forms. The $s k m 1$ isoform, expressed mostly in skeletal muscle, inactivates relatively quickly, and the $s k m 2$ isoform, present mostly in the myocardium and in developing or denervated skeletal muscle (Kallan et al., 1990, 1993), inactivates more slowly. Recently, homologs to both of these forms have been cloned in Sternopygus and detected in the electric organ using reverse transcriptase/PCR (G. Lopreato, personal communication). One possibility is that estrogen and androgen alter electrocyte sodium channel kinetics, and thereby the EOD pulse duration, by inducing the differential expression of these two kinetically distinct sodium channel isoforms. Mature female and estrogen-treated fish have a higher proportion of skm1-like channels, whereas mature male and androgen-treated fish express mostly skm2-like channels. We are currently testing this hypothesis in our laboratory.

\section{REFERENCES}

Alves-Gomes J, Orti G, Haygood M, Heiligenberg W, Meyer A (1995) Phylogenetic analysis of South American electric fishes (Order Gymnotiformes) and the evolution of their electrogenic system: a synthesis based on morphology, electrophysiology and mitochondrial sequence data. Mol Biol Evol 12:298-318.

Becker J, Breedlove M, Crews D (1992) Behavioral endocrinology. Cambridge, MA: MIT.

Boyle MB, MacLusky NJ, Naftolin F, Kaczmarek LK (1987) Hormone regulation of $\mathrm{K}^{+}$channel messenger RNA in rat myometrium during oestrus cycle and pregnancy. Nature 330:373-375.

Bullock TH, Heiligenberg W (1986) Electroreception. New York, Wiley.

Enger PS, Szabo T (1968) Effects of temperature on discharge rate of the electric organ of some gymnotids. Comp Biochem Physiol A. 27:625-627.

Erulkar SD, Rendt J, Nori RD, Ger B (1994) The influence of 17B oestradiol on $\mathrm{K}^{+}$currents in smooth muscle cells isolated from immature rat uterus. Proc Royal Soc Lond - Series B. 256:59-65.

Ferrari MB, Zakon HH (1993) Conductances contributing to the action potential of Sternopygus electrocytes. J Comp Physiol A. 173:281-292.

Ferrari MB, McAnelly ML, Zakon HH (1995) Individual variation and androgen modulation of the sodium current in electric organ. J Neurosci 15:4023-4032.

Fostier A, Jalabert B, Billard R, Breton B, Zohar Y (1983) The gonadal steroids. In: Fish physiology, Vol. 9B (Hoar WS, Randall DJ, Donaldson EM, ed), pp 277-372. New York: Academic Press.

Gustavson S, Zakon H, Prins G (1994) Androgen receptors in the brain, electroreceptors and electric organ of a wave-type electric fish. Soc Neurosci Abstr 20:371.

Hopkins CD (1974) Electric communication in the reproductive behavior of Sternopygus macrurus. Z. Tierpsychol 35:518-535.

Inoue Y, Sperelakis N (1991) Gestational changes in the sodium and calcium channel current densities in rat myometrial smooth muscle cells. Am J Physiol 260:C658-663.

Joels M, Karst H (1995) Effects of estradiol and progesterone on voltage- 
gated calcium and potassium conductances in rat CA1 hippocampal neurons. J Neurosci 15:4289-4297.

Kallan RG, Shen ZH, Yang J, Chen L, Rogart RB, Barchi RL (1990) Primary structure and expression of a sodium channel characteristic of denervated and immature rat skeletal muscle. Neuron 4:233-242.

Kallan RG, Cohen SA, Barchi RL (1993) Structure, function and expression of voltage- dependent sodium channels. Mol Neurobiol 7:383-428.

Kelley DB (1988) Sexually dimorphic behaviors. Ann Rev of Neurosci 11:225-251.

Kobayashi M, Stacey N (1993) Prostaglandin-induced female spawning behavior in goldfish (Carassias auratus) appears independent of ovarian influence. Horm Behav 278:38-55.

Lagrange AH, Ronnekleiv OK, Kelly MJ (1995) Estradiol-17B and muopiod peptides rapidly hyperpolarize GnRH neurons: a cellular mechanism of negative feedback? Endocrinol 136: 2341-2344.

Lam TJ (1983) Environmental influences on gonadal activity in fish. In: Fish physiology, Vol. 9A (Hoar WS, Randall DJ, Donaldson EM, ed) pp. 65-116. New York: Academic Press.

Liley NR, Stacey NE (1983) Hormones, pheromones and reproductive behavior in fish. In: Fish physiology, Vol. 9A (Hoar WS, Randall DJ, Donaldson EM, ed) pp 1-64. New York: Academic Press.

Liley NR, Wishlow WP (1974) The interaction of endocrine and experimental factors in the regulation of sexual behavior in the female guppy, Poecilia reticulata. Behavior 48:185-214.

McAnelly L, Zakon HH (1996) Protein kinase A activation increases sodium current magnitude in the electric organ of Sternopygus. J Neurosci 16:4383-4388.

Mermelstein PG, Becker JB, Surmeier DJ (1996) Estradiol reduces calcium currents in rat neostriatal neurons via a membrane receptor. J Neurosci 16:595-604.

Meyer JH (1983) Steroid influences upon the discharge frequencies of a weakly electric fish. J Comp Physiol A 153:29-37.

Meyer JH (1984) Steroid influences upon discharge frequencies of intact and isolated pacemakers of weakly electric fish. J Comp Physiol A 154:659-668.

Meyer JH, Leong M, Keller CH (1987) Hormone-induced and maturational changes in electric organ discharges and electroreceptor tuning in the weakly electric fish, Apteronotus. J Comp Physiol A 160:385-394.

Mills A, Zakon HH (1987) Coordination of the EOD frequency and pulse duration in a weakly electric wave fish: the influence of androgens. J Comp Physiol A 161:417-430.
Mills A, Zakon HH (1991) Chronic androgen treatment increases action potential duration in the electric organ of Sternopygus. J Neurosci 11:2349-2361.

Numann R, Catterall WA, Scheuer T (1991) Functional modulation of brain sodium channels by protein kinase $\mathrm{C}$ phosphorylation. Science 254:115-118.

Pakdel F, Feon S, Le Gac F, Le Menn F, Valotaire Y (1991) In vivo estrogen induction of hepatic estrogen receptor mRNA and correlation with vitellogenin mRNA in rainbow trout. Mol Cell Endocrinol 75:205-212.

Salbert G, Atteke C, Bonnec G, Jego P (1993) Differential regulation of the estrogen receptor mRNA by estradiol in the trout hypothalamus and pituitary. Mol Cell Endocrinol 96:177-82.

Schaefer JE, Zakon HH (1996) Opposing actions of androgen and estrogen on in vitro firing frequency of neuronal oscillators in the electromotor system. J Neurosci 16:2860-2868.

Stacey NE (1976) Effects of indomethacin and prostaglandins on spawning behavior of female goldfish. Prostaglandins 12:113-126.

White RE, Darkow DJ, Lang JL (1995) Estrogen relaxes coronary arteries by opening BKCa channels through a cGMP-dependent mechanism. Circ Res 77:936-42.

Yamamoto T (1995) Effects of estrogens on calcium channels in myometrial cells isolated from pregnant rats. Am J Physiol 268:C64-69.

Yasay GD, Kau ST, Li JH (1995) Mechanoinhibitory effects of estradiol on guinea pig urinary bladder smooth muscles. Pharmacol 51:273-80.

Zakon HH (1993) Weakly electric fish as model systems for studying long-term steroid action on neural circuits. Brain Behav Evol 42:242-251.

Zakon HH, Yan H, Thomas P (1990) Human chorionic gonadotropininduced shifts in the electrosensory system of the weakly electric fish, Sternopygus. J Neurobiol 21:826-833.

Zakon HH, Mills AC, Ferrari MB (1991a) Androgen-dependent modulation of the electrosensory and electromotor systems of a weakly electric fish. Seminars Neurosci 3:449-457.

Zakon HH, Thomas P, Yan H (1991b) Electric organ discharge frequency and plasma sex steroid levels during gonadal recrudescence in a natural population of the weakly electric fish Sternopygus macrurus. J Comp Physiol A 169:493-499. 